\title{
Orbit Correction Techniques for a Multipass Linac
}

\author{
A. Barry, B. Bowling, J. Kewisch, and J. Tang
}

Continuous Electron Beam Accelerator Facility

12000 Jefferson Avenue

Newport News, VA 23606

\section{Continuous}

\section{ELECTRON}

BEAM

ACCELERATOR

FACILITY

II0 $1 \% 1000$ 
Copies available from:

\author{
Library \\ CEBAF \\ 12000 Jefferson Avenue \\ Newport News \\ Virginia 23606
}

The Southeastern Universities Research Association (SURA) operaies the Continuous Electron Beam Acceierator Facility for the United States Department of Energy under contract DE-AC05-84ER40150.

\title{
DISCLAIMER
}

This report was prepared as an account of work oponsored by the United States government. Neither the United States nor the United States Department of Energy, nor any of their employees, makes any warranty, express or implied, or assumes any legal liability or responsibility for the accuracy, completeness, or usefulness of any information, apparatus, product, or process disclosed, or represents that its une would not infringe privately owned rights. Reference berein to any specific commercial product, process, or aervice by trade name, mark, manufacturer, or otberwise, does not necessarily constitute or imply its endorsement, recommendation, or favoring by the United States government or any agency thereof. The views and opinions of authors expressed berein do not necessarily state or reflect those of the United States government or any agency thereof. 


\title{
ORBIT CORRECTION TECHNIQUES FOR A MULTIPASS LINAC*
}

\author{
A. Barry, B. Bowling, J. Kewisch, and J. Tang \\ Continuous Electron Beam Accelerator Facility \\ 12000 Jefferson Avenue, Newport News, VA 23606
}

\begin{abstract}
The CEBAF accelerator includes a linac section which accelerates multiple beams with different energies. Problems arise when performing orbit correction, due to the fact that correction of higher energy passes disturb lower energy trajectories. Therefore, a perfect orbit correction cannot be obtained for all energy beams at the same time.
\end{abstract}

We present methods and performance (using simulation results) for performing orbit corrections in such a system. Limitations to the correction methods are also ad. dressed.

\section{Introduction}

The electron beam in the CEBAF accelerator is recirculated five times in order to make the most efficient use of the accelerating structure in the linac. Therefore, the orbit correction in the linacs is distinguished from other existing high energy inear accelerators by the fact that five beams, each with different energies, travel at the same time through the linac.

The linacs are equipped with beam position monitors, which have the ability to differentiate between the tive beams, and correction dipoles adjacent to eaci quadrupole. The orbit correction of the lowest energy beam can therefore be done simply by making the beam offset in each monitor zero, using the correctors attached to the previous quadrupole. However, these corrector settings are not necessarily the optimum settings for the higher energy passes. Therefore, a perfect orbit crsrection cannot be obtained simulaneousiy for all beams.

Most of the orbit distortions are caused by misplace. ment of quadrupoles $(\sigma=0.2 \mathrm{~mm}$ ) n.sd misalignment (pitch and yaw) of the linac cavities $(\sigma=2 \mathrm{mrad})$. Other smaller contributions come from steering due to the asymmetric RF feeds and residual magnetic fields of the earth's magnetic field. In addition to these active distortions, we assume that the beam position monitors have an accuracy of $0.1 \mathrm{~mm}$ and a misplacement relative to the quadrupole of $0.2 \mathrm{~mm}$.

All orbit correction schemes predict the effect of a corrector kick at one point on the beam position in monjtor at a second point from the strength of the quadrupoles between the two puints. In our simulation we rasumed a strength error of $\frac{\Delta k}{k}=10^{-3}$ for all quadrupoles.

All correction procedures described in this paper have been simulated using the computer code PETROS. ${ }^{1}$

* This work was supported by the U.S. Department of Energy under contract DE-AC05-84ER 40150.

\section{First Pass Correction}

The idea of the first pass correction is very simple: Since the simulated linac design uses an alternating beam position monitor and corrector pattern, we compute the upstream corrector value required to make a downstream monitor reading zero. The kjcks $\Delta x_{k}^{\prime}$ needed in the correc. tion coil is calculated from the beta functions and phase adrance:

$$
\Delta x_{k}^{\prime}=-\frac{\Delta x_{m}}{\sqrt{\beta_{k} \beta_{m}} \sin \left(\phi_{m}-\phi_{k}\right) \sqrt{\frac{p_{k}}{p_{m}}}}
$$

In this expression, $p_{k}$ and $p_{m}$ are the momenta of the electron beans in the corrector and the monitor, respectively, This factor must be included due to the energy gain in the cavities. For the same reason the beta functions have to be calculated according to reference 2. Figure 1 sbows the corrected low energy beam together with the higher energy passes.

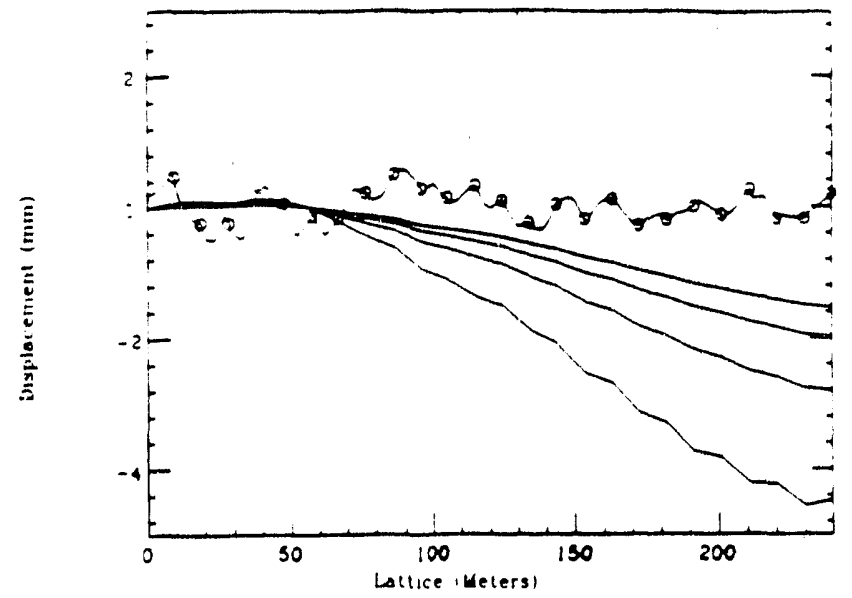

Figure 1. Low energy corrected orbit with higher energy passes.

\section{Correction of the Higher Passes}

Further correction of the higher passes through the use of correction coils is limited because any change applied to a corrector will disturb the low energy heam. The following methods can be implemented for the correction of the higher energies: (1) Optimization of injection angle and displacement, (2) the use of beam bumps in the low energy line, which act as a kick for the higher energies, (3) a combination of the above methods, (4) a least-squares fit to 26 variables ( 26 corrector dipoles with 26 monitor read. ings), and (5) 36 variables ( 26 correctors and 10 initial cin. ditions). 


\section{Optimization of the Initial Beam Coordinates}

The first correction step for the higher passes is the optimization of the angle and displacement for the injection of the beams into the linac. This is done with two correction coils immediately preceding the linac. While any displace. ment within the aperture can be produced in theory with these coils, the injection angle is limited by the beam pipe aperture. Figure 2 is the result of this correction.

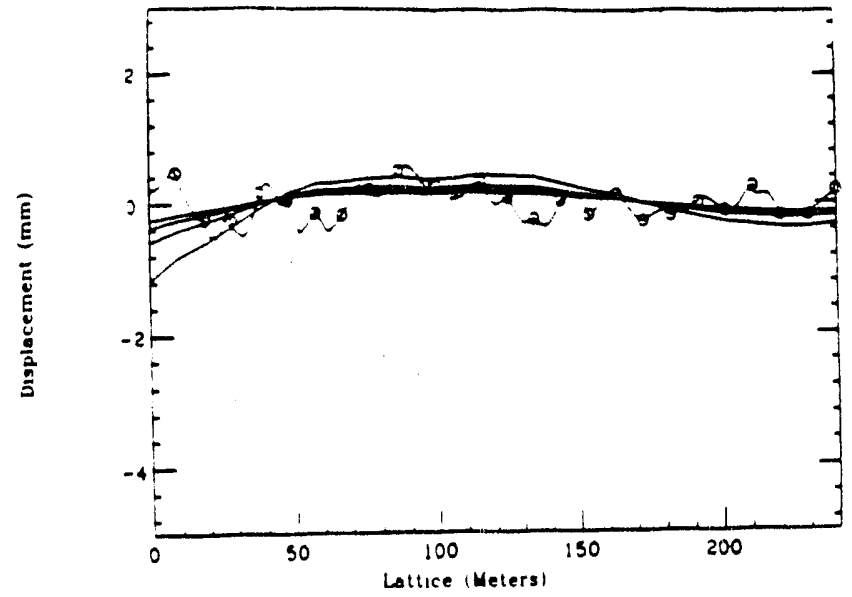

Figure 2. Optimization of injection paramelers.

\section{Correction Using Beam Bumps}

The idea of this correction method is to produce a beam bump in the lowest energy beam in order to produce a deflection of the higher energies that propagate down the linac. This has the effect of improving higher passes by sacrificing the orbit in a limited region of the lowest pass. With a bump of 2.5 mm in the low energy beam, this method corrects the higher passes ef effectively as the previous method.

\section{Least-Squares Fit Method}

This method determines values for all of the correctors simultaneously in an attempt to find the optimum orbits for all of the energy lines. The method is equivalent to applying simultaneously all possible bearn bumps. The result is better than the single beam bump method and also avoids the large excursion in the first energy line.

\section{Combination of Methods}

The combination of the beam bump method with simultaneous adjustment of the initial conditions gave a larger displacement of the first beam than in the case of the beam bump only.

The extension of the matrix method to include the initial conditions for each energy line in this method failed due to an ill-conditioned matrix. The failure with both attempts to combine correction methods indicates that this concept of method integration has inherent difficulties.
We have seen previously that each of the methods by themselves is comparably effective. When used in combi. nation, however, the methods tend to work against each other, resulting in a small improvement in orbit. The other correction schemes are therefore preferred.

\section{Systematic Misalignment Errors}

Systematic misalignment errors arise from the lower accuracy in measuring larger distances. We have simulated this case and have assumed a maximum sagitta of $5 \mathrm{~mm}$. The orbits after correction of the initial conditions are shown in Figure 3 for two fiducials.

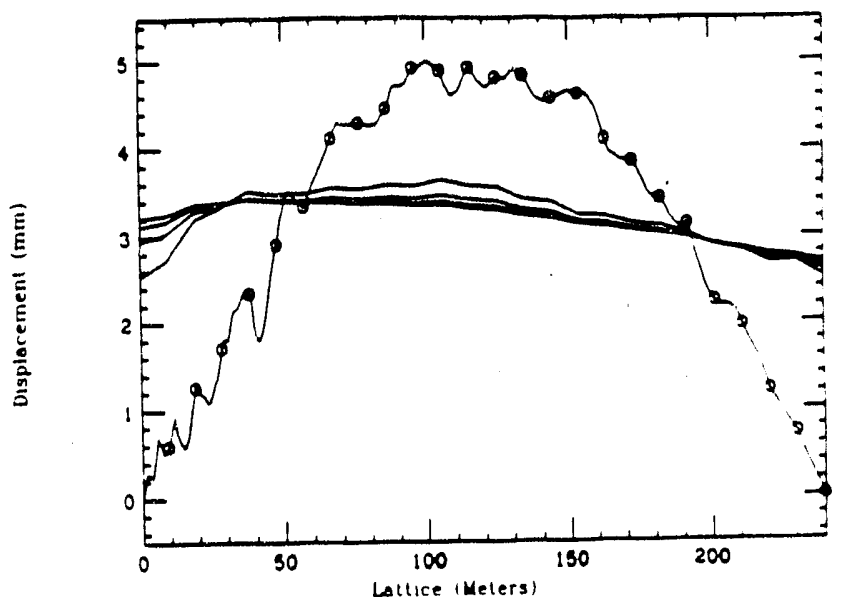

Figure 3. Systematic error with two fiducials.

We see that the highest energy beam is a "straight" line within $0.4 \mathrm{~mm}$. The high energy beam can therefore be used in both linacs to establish a reference line for the alignment of the linac. From the viewpoint of the beam position monitors, the high energy beam oscillates around the center of the quadrupoles. A good rule of thumb is that we can expect deviations of the high energy beams from the center of the quadrupoles of approximately half the linac sagitta.

\section{Limitations}

Three effects which provide an influence on the performance of the preceding orbit correction methods were investigated: (1) Changes in linac lattice lengths, (2) cavity operating gradient, and (3) betatron phase advance.

The above simulation results were obtained using a model of the CEBAF lattice. This linac has a designed length of 240 meters. In the future, it may be desirable to construct recirculating accelerating linacs of longer physical lengths. Therefore, to further test the abilities of the correction methods, a simulated linac was created with a length of 960 meters, 4 times the length of the CEBAF unit. The maximum orbit excursion exhibited when apply- 
ing beam entrance corrections is approximately the same as for the shorter linac. From this result it is apparent that longer accelerating sections are correctable using this method.

Cavity gradient clearly plays a role in the correction methods. Using the long linac model, cavity gradients were varied, and the maximum orbit deviations were noted. Figure 4 is a plot of these deviations as a function of gradient. From this, the predictable result is that the correction method is more effective for higher cavity gradients.

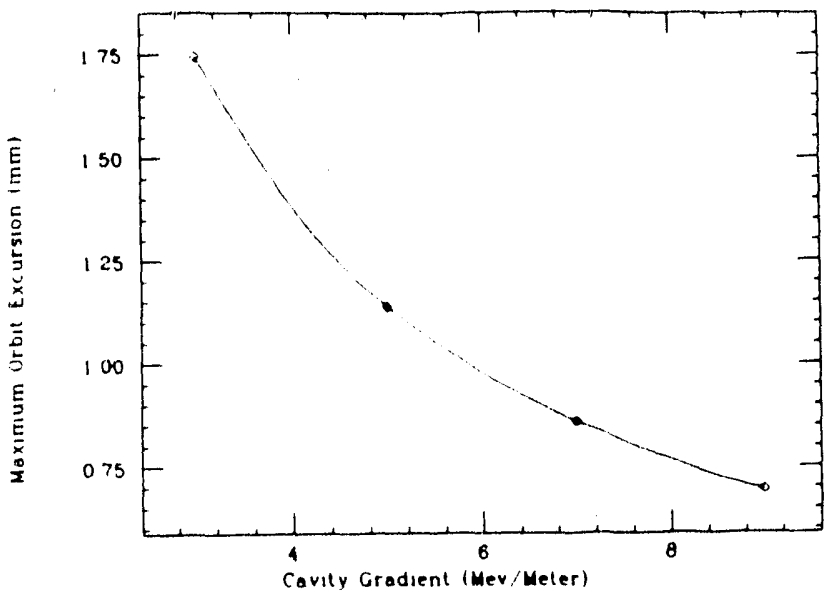

Figure 4. Carity gradient effects on orbit deviations.

A similar analysis was done for betatron phase advance (Figure 5). Here we see a small trend in orbit deviation with increasing phase advance. This effect is due mainly to the effect of quadrupole influences discussed earlier.

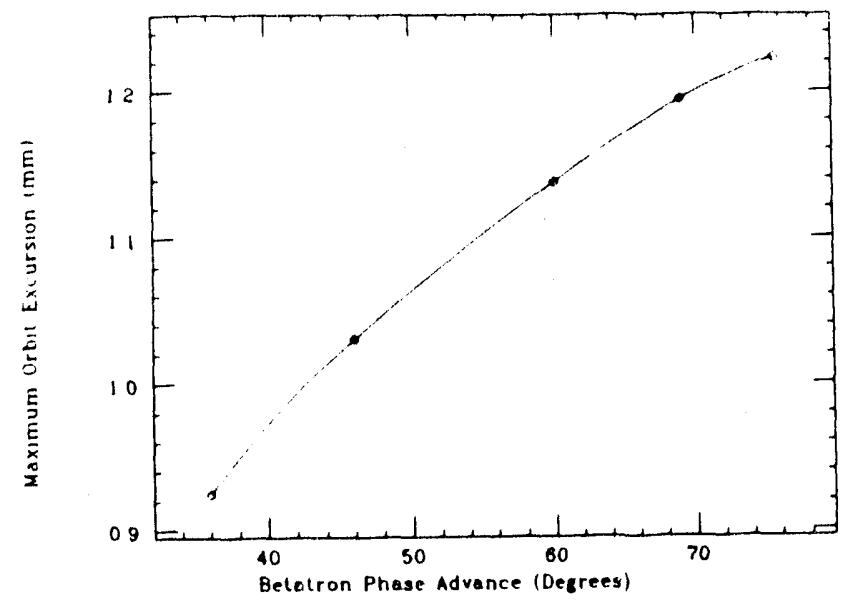

Figure 5. Betatron phase advance effects on orbit deviations.

\section{References}

1. K. Steffen, J. Kewisch: Study of Integer Difference Resonance in Distorted PETRA Optics, DESY Internal Report (1976).

2. D. Douglas, J. Kewisch, R. Fork: Betatron Function Parameterization of Beam Optics Including Acceleration, CEBAF-PR-89-0!1, Proc. 1988 Linac Conference. 

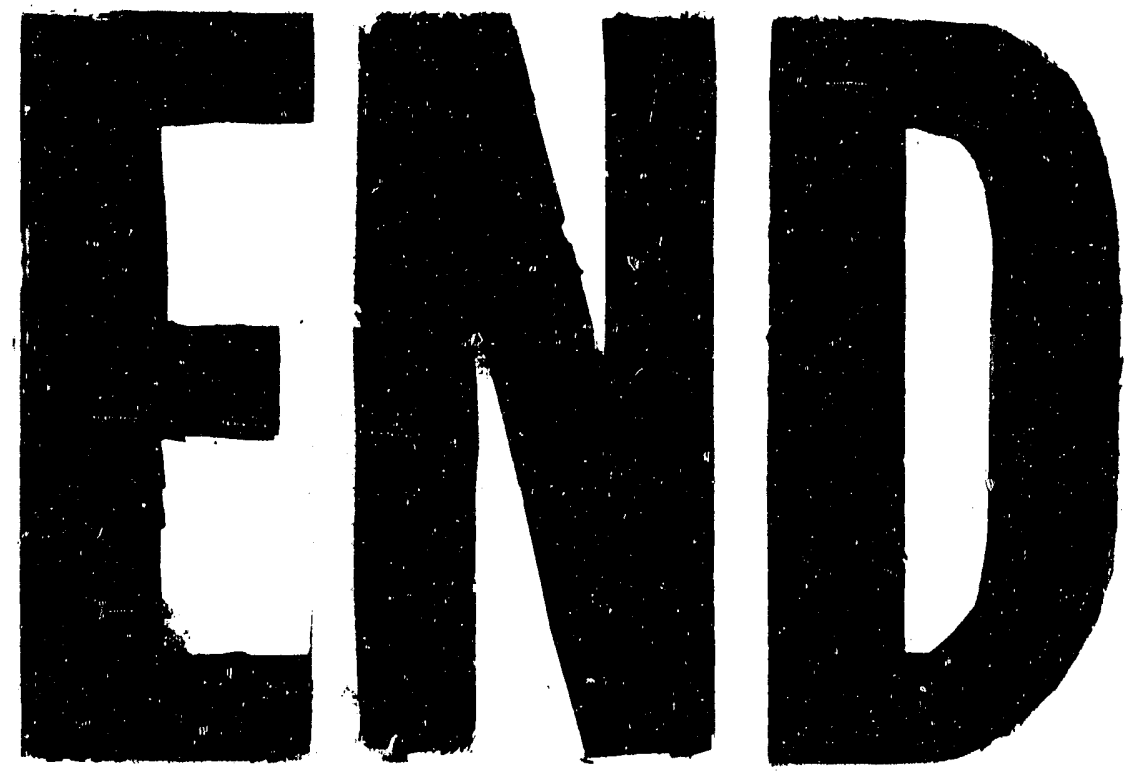

5
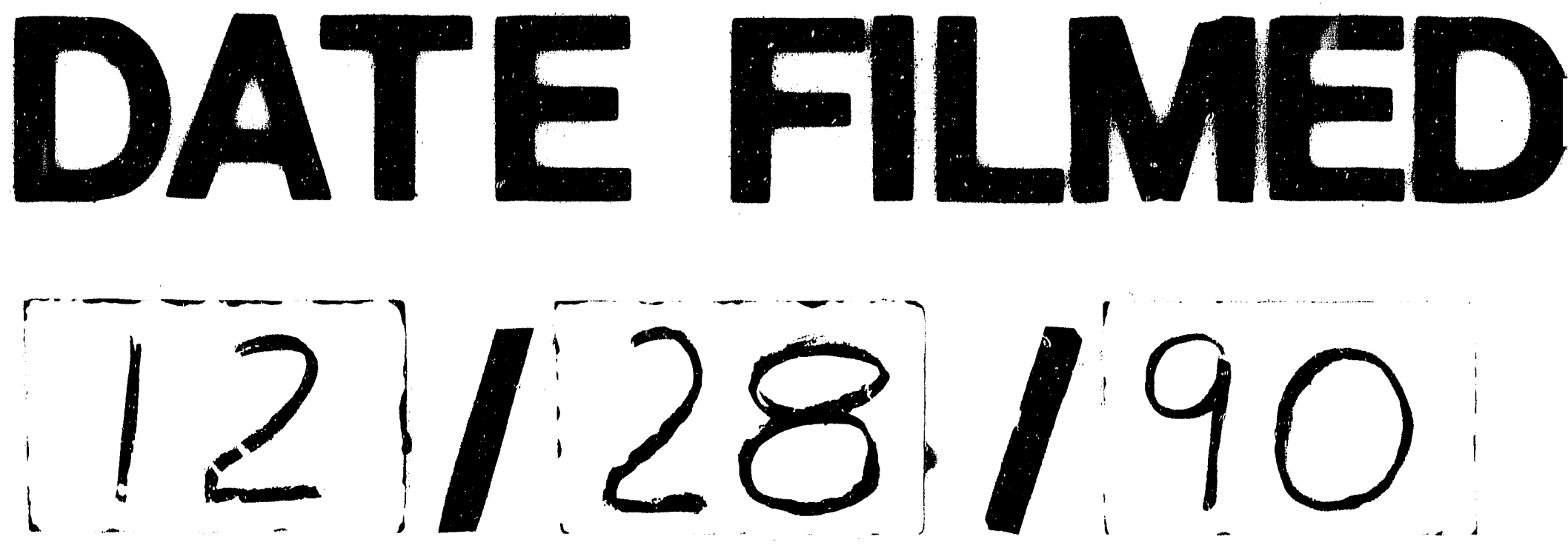
